\title{
Scoliosis, CTCAE
}

National Cancer Institute

\section{Source}

National Cancer Institute. Scoliosis, CT CAE. NCI Thesaurus. Code C143824.

A disorder characterized by a malformed, lateral curvature of the spine. 